\title{
Comparing Lifestyle Convenience of Multiple Daily Injection and Continuous Subcutaneous Insulin Infusion
}

\author{
Ikuko Nishio \\ Department of Adult Nursing, Faculty of Nursing, Soka University, Hachioji City, Japan \\ Email: nishio@soka.ac.jp
}

How to cite this paper: Nishio, I. (2017) Comparing Lifestyle Convenience of Multiple Daily Injection and Continuous Subcutaneous Insulin Infusion. Open Journal of Nursing, 7, 1365-1374.

https://doi.org/10.4236/ojn.2017.712098

Received: October 26, 2017

Accepted: December 5, 2017

Published: December 8, 2017

Copyright $\odot 2017$ by authors and Scientific Research Publishing Inc. This work is licensed under the Creative Commons Attribution International License (CC BY 4.0).

http://creativecommons.org/licenses/by/4.0/

Open Access

\begin{abstract}
Aim: This study investigated the lifestyle convenience of Insulin therapy for adult women patients with type 1 diabetes. Methods: Participants were type 1 diabetes adult women patients who switched from multiple daily injection (MDI) therapy to Continuous Subcutaneous Insulin Infusion (CSII) therapy. We conducted semi-structured interviews with participants. To analyze, we referenced the classification table of Hamada's study comparing the usefulness of both the NovoPen ${ }^{\oplus}$ and CSII. Semi-structured interviews were conducted with the research subjects. The questions focused mainly on diabetes management, instrument operation, everyday life, and social, psychological and lifestyle convenience aspects during MDI and CSII. Results: Research subjects were 4 women patients with type 1 diabetes. The average age was $35.3 \pm 6.24$ (SE) years old. Participants were undergoing MDI therapy; however, in the middle, they switched to CSII therapy. CSII therapy is convenient in terms of diabetes management, social and psychological. MDI therapy is convenient in terms of instrument operation and daily life. Compared to MDI therapy, CSII therapy is less convenient in terms of instrument operation and everyday life. However, since CSII therapy has more stable HbA1c and blood glucose levels compared to MDI therapy, it is more convenient in terms of social and psychological aspects and reduces those burdens. About economy CSII therapy, patients paid about 5000 yen more per month compared to MDI therapy. Conclusion: It is believed that patients prioritize therapeutic effects, and choose CSII, which is stable in psychological and social aspects, even though it is inconvenient with life.
\end{abstract}

\section{Keywords}

Insulin Infusion Systems, Lifestyle, Disease Management, Multiple Daily

Injection, Type 1 Diabetes, Women's Health 


\section{Introduction}

For type 1 diabetes treatment, there is Multiple Daily Injection (MDI) therapy and Continuous Subcutaneous Insulin Infusion (CSII) therapy. Recently, insulin pump improvement has progressed, and type 1 diabetes patients introduced to CSII therapy are increasing. In the Diabetes Control and Complication Trial [1], reported in 1998, the effectiveness of CSII therapy was reported. Since the amount of insulin can be adjusted to match the patient's lifestyle, the greatest benefit of CSII therapy is that it stabilizes fluctuations in blood glucose and can reduce the risk of severe hypoglycemia [2]-[6]. For Japanese CSII therapy subjects, it can be adapted for type 1 diabetes patients for whom glycemic control was poor in MDI therapy, as well as for patients who are pregnant or wish to be pregnant and give birth. In Japan, in April 2010, it became possible to receive treatment with health insurance as long as the medical institution met certain conditions; this made it even easier for type 1 diabetes patients to become introduced to CSII therapy, and it became possible to maintain favorable glycemic control over long periods [7] Currently, CSII therapy is recommended for type 1 diabetes therapy. However, no matter how highly effective the treatment, it has some influence on the patient's lifestyle. Depending on treatment instructions, limitations are applied to the patient's actions, and therefore, it becomes necessary to spend time and effort adapting to the lifestyle changes.

There are advantages and disadvantages to both CSII therapy and MDI therapy, and there has been much debate about which treatment is better for type 1 diabetes patients. However, it is difficult to determine which is superior [8] [9]. Not only are the features of CSII and MDI therapy different, but depending on the patient's individual lifestyle, the recognition of the treatment also differs. This is perhaps why lifestyle convenience for the patient is considered to be important. It is necessary to understand the differences in lifestyle convenience of both MDI and CSII therapy, and to investigate the necessary nursing for patients.

In this paper, we report on our comparative examination of instrument operation, diabetes management, everyday life, and social, and psychological aspects based on 4 cases of type 1 diabetes female patients who experienced both MDI and CSII therapy.

\section{Subjects and Methods}

\subsection{Participants}

From the adult type 1 diabetes patients visiting the diabetes internal medicine outpatient clinic, we selected patients who had received both MDI and CSII therapy. To compare the usefulness of both MDI and CSII therapy, we excluded patients with dementia, mental illness, or severe complications (nephropathy undergoing dialysis, lower limb amputation, or blindness.

\subsection{Data Collection}

With the permission of the physician in charge of the research subjects, we used 
the open hours of the outpatient clinic (about 1 hour between blood collection and examination), and conducted semi-structured interviews in one corner of the waiting room. For the interview, patients spoke freely about instrument operation, diabetes management, everyday life, social aspects, and psychological aspects during the times they received MDI and CSII therapy. With the research subjects' consent, interviews (each of which lasted for 60 - 75 minutes) were recorded with an IC recorder.

\subsection{Data Analysis}

For data analysis, we created a verbatim record of the content obtained from the semi-structured interviews. We extracted descriptions considered to express the research subjects' thoughts on lifestyle convenience while undergoing MDI and CSII therapy. To analyze lifestyle convenience in detail, we referenced the classification table of Hamada's [10] study comparing the usefulness of both the NovoPen $^{\oplus}$ and CSII and decided to look at lifestyle convenience from 5 aspects, diabetes management ( 5 items), instrument operation ( 2 items), everyday life (8 items), social aspect ( 3 items), and psychological aspect ( 2 items). We examined the meaning of extracted data, consolidated content with similar meaning, and classified each item. Through the analysis process, to arrive at the appropriate interpretation, data was examined until consistency of opinion was achieved among co-researchers, and clarity and potential reliability was ensured. We relayed the findings to the participants as well, and were able to obtain their views.

\subsection{Ethical Considerations}

This study was approved by the Ethics Committee of the Faculty of Medicine, Tottori University. Informed consent was obtained from all of the patients using a procedure approved by the Ethics Committee. We explained to research participants the study's purpose, free will of participation in the study, privacy considerations, and the right to withdraw from participation at any time during the study. We obtained consent both in writing and orally. For the interview location, we took into consideration a location in which other people would not be able to hear the interview content. Interviews were recorded with the subjects' understanding.

\section{Results}

\subsection{Overview of Research Subjects}

Overview of the research subjects is shown in Table 1. Research subjects were 4 women with type 1 diabetes. The average age was $35.3 \pm 6.24$ (SE) years old, and average disease duration was $7.3 \pm 3.8$ years (SE). Research subjects were undergoing MDI therapy; however, in the middle, they switched to CSII therapy, because CSII had the advantage of infusing insulin continuously into the participants' body. 
Table 1. Demographic characteristics of the 4 study participants with type 1 diabetes.

\begin{tabular}{|c|c|c|c|c|}
\hline Items & Patient A & Patient B & Patient C & Patient D \\
\hline Age & 29 & 33 & 39 & 42 \\
\hline Gender & Female & Female & Female & Female \\
\hline Occupation & Housewife & Housewife & Care worker & Contracted worker \\
\hline Time living with Type 1 diabetes (years) & 4 years & 10 years & 11 years & 4 years \\
\hline CSII introduction period (years) & 3 years & 0.5 years & 8 years & 1 years \\
\hline MDI introduction period (years) & 1 year & 9.5 years & 3 years & 3 years \\
\hline Blood glucose levels & $100-150 \mathrm{mg} / \mathrm{dl}$ & $200-250 \mathrm{mg} / \mathrm{dl}$ & $130-150 \mathrm{mg} / \mathrm{dl}$ & $120-160 \mathrm{mg} / \mathrm{dl}$ \\
\hline $\begin{array}{l}\text { Location where the patient received } \\
\text { the insulin injection (MDI) }\end{array}$ & Home & Home & $\begin{array}{c}\text { Home and the restroom } \\
\text { of her office }\end{array}$ & $\begin{array}{c}\text { Home and the restroom } \\
\text { of her office }\end{array}$ \\
\hline HbAlc (NGSP) prior to CSII introduction & The $9 \%$ first half & The $9 \%$ first half & The $9 \%$ first half & The 7\%last half \\
\hline HbAlc (NGSP) after CSII introduction & The $7 \%$ first half & The $8 \%$ first half & The $7 \%$ last half & The $7 \%$ first half \\
\hline $\begin{array}{l}\text { Reasons for switching from } \\
\text { MDI therapy to CSII therapy }\end{array}$ & $\begin{array}{l}\text { Due to pregnancy, } \\
\text { was recommended } \\
\text { by a doctor to } \\
\text { switch. }\end{array}$ & $\begin{array}{c}\text { Due to the desire to } \\
\text { become pregnant, } \\
\text { consulted with a } \\
\text { doctor and } \\
\text { switched to CSII. }\end{array}$ & $\begin{array}{l}\text { Could not inject during } \\
\text { work and there were many } \\
\text { incidences of hypoglycemia, } \\
\text { so desired to switch to CSII. }\end{array}$ & $\begin{array}{l}\text { Was recommended } \\
\text { by a doctor since } \\
\text { HbAlc levels } \\
\text { did not drop with } \\
\text { insulin injections. }\end{array}$ \\
\hline Complication of diabetes & No & No & No & No \\
\hline
\end{tabular}

HbAlc: Glycated hemoglobin. NGSP: National Glycohemoglobin Standardization Program. CSII: Continuous subcutaneous insulin infusion: MDI: Multiple daily injection.

\subsection{Diabetes Management}

Patients in CSII therapy experienced skin trouble such as "marks remain after the injection" and "sometimes rashes appear". In MDI therapy as well, there were skin troubles such as "the injection site becomes blue" and "the skin becomes hard if I inject at the same location". In terms of alleviating pain, there were experiences in CSII therapy such as "If the puncture location is bad, there is pain every time the medication enters", while in MDI therapy, there were experiences such as "Since I am right-hand dominant, when I inject the left side of the abdomen, the needle is at an angle and sometimes it is painful". Although pain was experienced in both therapies, pain was more minor with MDI therapy.

In CSII therapy, HbAlc and blood glucose levels were stable and numerical values improved since insulin was being injected regularly, with experiences such as "HbAlc was stable and improved" and "I pay attention to food amounts since blood glucose levels rise when carbohydrates are ingested". In MDI therapy, HbAlc and blood glucose levels were unstable and when injections were not done during work, this caused incidences of both hypo and hyperglycemia, with experiences such as "HbA1c is always high", "Sometimes I do not inject during work", "Since it is unpleasant to go to the bathroom and inject, sometimes I do not inject", "Fluctuations in blood glucose levels were large", and "There were 
many times during work when I did not measure my blood glucose level even though I wanted to". For hypoglycemia, in CSII therapy, there were experiences such as "There is low frequency of hypoglycemia because the amount of additional insulin (bolus) can be adjusted" and "If too much bolus is taken, one can become hypoglycemic". In MDI therapy, there were experiences such as "since there are times when I skip a meal during work, I often become hypoglycemic". Compared to MDI therapy, a decrease in frequency of hypoglycemia was seen with CSII therapy. However, with both therapies, patients were devising ways to not become hypoglycemic.

\subsection{Instrument Operation}

With CSII therapy, 1 needle injection per 6 days is adequate while with MDI therapy, daily injections are necessary, and therefore CSII therapy is more convenient. Ease of instrument operation with CSII therapy is "Difficult" and "Takes time to get used to", and troubles include "Injections are not performed and the tube gets clogged". However, with MDI therapy, instrument operation is "Easy", "Sometimes I forget to inject it", and there was "No trouble, not even once". For ease of instrument operation, MDI therapy is more convenient than CSII therapy in that there was no trouble with instrument operation; however, forgetting to inject was directly related to risk of hyper or hypoglycemia.

\subsection{Daily Life}

Regarding ease of cooking, ease of cleaning, ease of child care, ease of bathing, quality of sleep, freedom of clothing, and ease of using the toilet, lifestyle convenience was poor with CSII therapy due to wearing the pump and having the puncture site in the abdomen, with experiences such as "There is pain when the puncture location hits the kitchen counter", "Does not feel tired", "Cannot wipe and clean while squatting", "Depending on the insertion location, it might hit the child's leg when you a hug a child, and it may fall off", "Since it is not waterproof, I remove the insulin pump", "Since the pump gets in the way, I move it without realizing it (moving while sleeping)", "The insulin pump will stick out if I don't wear loose clothing", and "Since people dress lightly in summer, clothing is particularly limited". With MDI therapy, convenience was poor for ease of cleaning and freedom of clothing, with experiences such as "I tire easily", "I take breaks while cleaning", and "I cannot wear a dress, but compared to with an insulin pump, I can choose clothes more freely". However, convenience was better regarding ease of cooking, ease of child care, ease of bathing, quality of sleep, and ease of using the toilet. Regarding ease of going out/eating out, CSII therapy had good convenience, with experiences such as "When eating out, I can do the injection there". MDI therapy, on the other hand, had poor convenience for eating out due to having to find and secure a place to inject, with experiences such as "it is difficult to inject in front of people", "I have trouble finding a place", and "I have at times forgotten the injection equipment when I went out". 


\subsection{Social Aspects}

In both CSII therapy and MDI therapy, patients felt restrained, with experiences in CSII therapy such as "Until you get used to it, the insulin pump is annoying", and in MDI therapy, "I am always searching for a place to inject" and "I have to walk around with injection equipment".

With CSII therapy, work efficiency was better and convenience was good as a result of stable blood glucose levels, with experiences such as "I am now able to work thanks to switching to an insulin pump" and "I can set up a base according to the work". MDI therapy had poor convenience due to not being able to inject in a clean place and taking time to inject insulin during work, and there were experiences such as "At work, I inject in the bathroom" and "I cannot work if I become hypoglycemic". CSII therapy cost around 15,000 to 20,000 yen per month, and MDI therapy cost around 10,000 yen per month. For CSII therapy, patients paid about 5000 yen more per month compared to MDI therapy.

\subsection{Psychological Aspects}

Both therapies influence body image, with CSII therapy experiences such as "I have to make sure that you can't see the insulin pump" and "The burden of doing 4 to 5 injections per day decreased", and for MDI therapy, there were experiences such as "I cannot inject in front of people" and "I tell children not to tell surrounding people that I am injecting". However, regarding a sense of burden, with CSII therapy, blood glucose levels are stable and there is decreased burden of having to do multiple injections, so patients felt a return to psychological stability, with experiences such as "Blood glucose levels were stable and I was mentally stable", "Blood glucose level worries have decreased", and "the burden of doing 4 to 5 injections per day decreased". However, with MDI therapy, there was a sense of burden as a result of having to do multiple injections daily and large fluctuations in blood glucose levels, with experiences such as "It is psychologically difficult to do multiple daily injections" and "Stress accumulates because blood glucose levels and HbA1c are not stable".

\section{Discussion}

\subsection{Lifestyle Convenience of CSII and MDI Therapy for Type 1 Diabetes}

In this study, all research subjects were female and had to take on both housework and child care duties. Compared to men, they had a high interest in personal appearance, and it was clear that lifestyle convenience was important to them. It is reported that patients in CSII therapy feel uncomfortable with the constant weight of the insulin pump and having the pump on their abdomen all day. ${ }^{9}$ With CSII therapy, they are always wearing the insulin pump. Therefore, it became clear that the pump itself impacts housework behavior and lifestyle behavior and is a factor intervening with convenience. Particularly for child care convenience, the younger the child is, the more time child care requires and the 
more the child gets picked up. While taking care of a child, it is necessary to consider not only the CSII therapeutic effects, but also how to not let it infere with child care. Similarly, for freedom of clothing, women not only have to wear the pump and choose clothes accordingly, but they also devise ways so the pump does not show, and they must be fashionable. Regarding ease of cooking and cleaning, based on patients' stories, we assume that female patients require convenience as a factor. There are no research papers clarifying the lifestyle experiences of female patients during CSII therapy; however, women take on roles of wife, daughter-in-law, and mother, and we presume that they require lifestyle flexibility. To patients, the pros and cons of CSII convenience influence blood glucose control. Therefore, for female patients, considering housework and child care responsibilities, it is necessary to keep lifestyle convenience in mind.

In terms of MDI therapy convenience, the fact that a patient must leave her seat to go inject and must secure a place to inject negatively influences lifestyle convenience. These are also thought to influence diabetes management. Not being able to inject in front of people and having to find a place to inject lead to poor behavior such as not being able to inject or not injecting. As a result, blood glucose levels and HbA1c worsen and there are frequent incidences of hypoglycemia, and this leads to a worsening of diabetes. In terms of social aspects, encountering both a sense of burden to inject insulin and not being able to inject influenced work and led to psychological difficulties. Garmo [11] reports that the more long-term MDI therapy is, the greater the psychological burdens become. Similarly, this research also indicates that since with MDI therapy, diabetes management is not stable, MDI therapy impacts a psychological sense of restraint and burden and social aspects of work and finances. However, in terms of convenience for housework behavior and lifestyle behavior in everyday life, MDI therapy was more convenient than CSII therapy. However, convenience was poor for ease of cleaning, which has a high amount of activity, and this is presumed to be caused by the severe changes in blood glucose levels when activity levels are high.

CSII therapy has poor convenience for instrument operation and everyday life. However, since it is more favorable than MDI therapy in terms of diabetes management, it can positively impact psychological and social aspects. We presume that patients will prioritize treatment effects, even if lifestyle convenience is poor, and so they will choose CSII, in which the psychological and social aspects are stable.

The medical costs of CSII therapy are higher than MDI therapy. However, since blood glucose levels and HbAlc are lowered and frequency of hypoglycemia decreases, it is possible to have improved blood glucose control with CSII therapy, and therefore, it has been reported that treatment satisfaction levels are high. [12] Diabetes management improves with CSII therapy, and therefore, and for social aspects, it becomes possible to continue work, and for psychological aspects, the burden of injecting multiple times per day and worries about hypoglycemia are reduced. Thus, CSII therapy not only improves diabetes man- 
agement, but good convenience for psychological and social aspects lead to continued treatment. It is also reported that regaining self-management through CSII therapy leads to benefits in QOL [13] [14] [15].

Thus, this study's research subjects were 4 cases continuing the transition from MDI therapy to CSII therapy. Although CSII therapy has poor convenience for female patients, it was clear that it was favorable not only for diabetes management, but also for psychological and social aspects. In a meta-analysis of CSII therapy, it is reported that compared to MDI therapy, blood glucose control for type 1 diabetes patients improves, although it does not improve for type 2 diabetes patients. ${ }^{5}$ Since this study's subjects were type 1 diabetes patients, this study's results show improvement in blood glucose control with CSII therapy and we presume that treatment will be continued. In regards to lifestyle convenience with instrument operation, everyday life, and social and psychological aspects, we understood that this study's patients tried various methods and experiences to avoid worsening blood glucose control. This study's results were similar to Rasmussen's [16] results stating that type 1 diabetes patients devise strategies utilizing past experiences to stablizie blood glucose levels. The more long term type 1 diabetes patients' insulin treatment is, the more troublesome it becomes and the more it leads to feelings of burden and restraint [17]. This is not limited to CSII therapy. However, the discomfort and hassle of attaching an insulin pump to one's abdomen 24 hours a day, 365 days per year is immeasurable. Patients prioritize the therapeutic effect, and therefore, it is important to work on everyday lifestyle convenience to make living easier for patients.

\subsection{Nursing Suggestions}

In this study, many patients prioritized lifestyle convenience as they underwent treatment while continuing to lead their everyday lives. Since this study's subjects were female, lifestyle conveniences such as ease of housework and child care, lack of skin trouble, and positive body image was essential. CSII therapy had a high therapeutic effect, yet poor lifestyle convenience [18], and MDI therapy had better lifestyle convenience than CSII therapy, yet had poor convenience in terms of therapeutic effects being unstable and not being able to inject in front of people. However, even though lifestyle convenience may be poor and troublesome for female patients, patients spoke of lifestyle convenience for both MDI and CSII therapy improving with nurses' support. Most nurses are female and often serve as guides on lifestyle conveniences unique to women. However, since individuals perceptions of lifestyle conveniences differ, it is necessary for nurses to have diabetes nursing experience so they can understand diabetes self-management and lifestyle conveniences. Even when nurses are busy with outpatient work, rather than focus on the physical aspects, it is important that they are aware of lifestyle convenience issues resulting from treatment, that they ask about questions, uncertainties, and worries, and that they understand how CSII and MDI therapy interfere with patients' everyday life. Additionally, it is important that this not be completed simply through conversation, but that pamphlets 
and checklists are made containing important and necessary information and continual guidance is given. By providing methods to help patients live comfortably, nurses can prevent treatment interruption or abandonment, and it is presumed that this will also lead to support for a favorable state of diabetes management. Even when nurses are busy, if they do not acquire the ability to listen to patients and explain to patients, they will not be able to guide patients toward an understanding or resolution of the factors interfering with their lifestyle convenience. Therefore, it is essential for nurses to build a relationship with patients so they can understand step-by-step what troubles the patient and what they find difficult, and to build communication skills.

\section{Study Limitations}

This study had 4 cases. Therefore, we cannot rule out that lifestyle convenience bias may have appeared. In the future, analysis of an increased number of cases is necessary.

\section{Acknowledgements}

We thank two patients who participated in this study for their contribution and dedication.

\section{References}

[1] Kilpatrick, E.S., Rigby, A.S. and Atkin, S.L. (2007) Insulin Resistance, the Metabolic Syndrome, and Complication Risk in Type 1 Diabetes: "Double Diabetes" in the Diabetes Control and Complications Trial. Diabetes Care, 30, 707-712. https://doi.org/10.2337/dc06-1982

[2] Weissberg-Benchell, J., Antisdel-Lomaglio, J. and Seshadri, R. (2003) Insulin Pump Therapy: A Meta-Analysis. Diabetes Care, 26, 1079-1087. https://doi.org/10.2337/diacare.26.4.1079

[3] Retnakaran, R., Hochman, J., DeVries, J.H., Hanaire-Broutin, H., Heine, R.J., Melki, V. and Zinman, B. (2004) Continuous Subcutaneous Insulin Infusion versus Multiple Daily Injections: The Impact of Baseline A1c. Diabetes Care, 27, 2590-2596. https://doi.org/10.2337/diacare.27.11.2590

[4] Hirsch, I.B., Bode, B.W., Garg, S., Lane, W.S., Sussman, A., Hu, P., Santiago, O.M. and Kolaczynski, J.W. (2005) Insulin Aspart CSII/MDI Comparison Study Group. Continuous Subcutaneous Insulin Infusion (CSII) of Insulin Aspart versus Multiple Daily Injection of Insulin Aspart/Insulin Glargine in Type 1 Diabetic Patients Previously Treated with CSII. Diabetes Care, 28, 533-538.

https://doi.org/10.2337/diacare.28.3.533

[5] Jeitler, K., Horvath, K., Berghold, A., Gratzer, T.W., Neeser, K., Pieber, T.R. and Siebenhofer, A. (2008) Continuous Subcutaneous Insulin Infusion versus Multiple Daily Insulin Injections in Patients with Diabetes Mellitus: Systematic Review and Meta-Analysis. Diabetologia, 51, 941-951. https://doi.org/10.1007/s00125-008-0974-3

[6] Pickup, J.C. (2012) Insulin-Pump Therapy for type 1 Diabetes Mellitus. The New England Journal of Medicine, 366, 1616-1624.

https://doi.org/10.1056/NEJMct1113948 
[7] Nishio, I., Chujo, M., Ohkura, T. and Kataoka, H. (2015) Opinions and Satisfaction regarding Continuous Subcutaneous Insulin Infusion Therapy in Adult Patients with type 1 Diabetes. Yonago Acta Medica, 58, 101-107.

[8] Bak, J.F., Nielsen, O.H., Pedersen, O. and Beck-Nielsen, H. (1987) Multiple Insulin Injections Using a Pen Injector versus Insulin Pump Treatment in Young Diabetic Patients. Diabetes Res, 6, 155-158.

[9] Saarinen, T., Fernström, L., Brorsson, A.-L. and Olinder, A.L. (2014) Insulin Pump Therapy Is Perceived as Liberating, But to Many It Can Imply a Sense of the Diabetes Made Visible. European Diabetes Nursing, 11, 38-42. https://doi.org/10.1002/edn.246

[10] Hamada, Y., Yoshida, N., Shimozawa, K., Ohta, Y., Fujiki, M. and Takazakura, E. (1992) Switch from Nobo Pen ${ }^{R}$ to CSII. Practice, 9, 164-166.

[11] Garmo, A., Garmo, H., Ärnlöv, J. and Leksell, J. (2011) Long-Term Treatment Effects of Insulin Pump Therapy. Practical Diabetes, 28, 295-299. https://doi.org/10.1002/pdi.1618

[12] Nishio, I. and Chujo, M. (2015) Type 1 Diabetes Patients Using Continuous Subcutaneous Insulin Infusion Therapy: Feeling Burdened Correlated with Factors. Yonago Acta Medica, 58, 123-128.

[13] Shwide-Slavin, C. (2003) Case Study: A Patient with Type 1 Diabetes Who Transitions to Insulin Pump Therapy by Working with an Advanced Practice Dietitian. Diabetes Spectrum, 16, 37-40. https://doi.org/10.2337/diaspect.16.1.37

[14] Ritholz, M.D., Smaldone, A., Lee, J., Castillo, A., Wolpert, H. and Weinger, K. (2007) Perceptions of Psychosocial Factors and the Insulin Pump. Diabetes Care, 30, 549-554. https://doi.org/10.2337/dc06-1755

[15] Barnard, K.D. and Skinner, T.C. (2008) Cross Sectional Study into Quality of Life Issues Surrounding Insulin Pump Use in Type 1 Diabetes. Practical Diabetes International, 25, 194-199.

[16] Rasmussen, B., Ward, G., Jenkins, A., King, S.J. and Dunning, T. (2011) Young Adults' management of type 1 diabetes during life transitions. Journal of Clinical Nursing, 20, 1981-1992. https://doi.org/10.1111/j.1365-2702.2010.03657.x

[17] Neylon, O.M., O’Connell, M.A., Skinner, T.C. and Cameron, F.J. (2013) Demographic and Personal Factors Associated with Metabolic Control and Self-Care in youth with Type 1 Diabetes: A Systematic Review. Diabetes/Metabolism Research and Reviews, 29, 257-227. https://doi.org/10.1002/dmrr.2392

[18] Braeger, J. (2011) Type 1 Diabetes Self-Management: A Patient's Experience of Using CSII. European Diabetes Nursing, 8, 17. https://doi.org/10.1002/edn.168 